PROCEEDINGS OF THE

AMERICAN MATHEMATICAL SOCIETY

Volume 127, Number 12, Pages 3467-3474

S 0002-9939(99)04943-6

Article electronically published on May 13, 1999

\title{
THE $K$-HOMOLOGY CLASS OF THE EULER CHARACTERISTIC OPERATOR IS TRIVIAL
}

\author{
JONATHAN ROSENBERG
}

(Communicated by Józef Dodziuk)

\begin{abstract}
On any manifold $M^{n}$, the de Rham operator $D=d+d^{*}$ (with respect to a complete Riemannian metric), with the grading of forms by parity of degree, gives rise by Kasparov theory to a class $[D] \in K O_{0}(M)$, which when $M$ is closed maps to the Euler characteristic $\chi(M)$ in $K O_{0}(\mathrm{pt})=\mathbb{Z}$. The purpose of this note is to give a quick proof of the (perhaps unfortunate) fact that $[D]$ is as trivial as it could be subject to this constraint. More precisely, if $M$ is connected, $[D]$ lies in the image of $\mathbb{Z}=K O_{0}(\mathrm{pt}) \rightarrow K O_{0}(M)$ (induced by the inclusion of a basepoint into $M$ ).
\end{abstract}

Let $M^{n}$ be a complete Riemannian manifold without boundary (possibly compact, possibly non-compact). Recall that the de Rham operator $D=d+d^{*}$, acting on differential forms on $M$ (of all possible degrees) is a formally self-adjoint elliptic operator, and that on the Hilbert space of $L^{2}$ forms, it is essentially self-adjoint [Ga]. With a certain grading on the form bundle (coming from the Hodge $*$-operator), $D$ becomes the signature operator; with the more obvious grading of forms by parity of the degree, $D$ becomes the Euler characteristic operator. When $M$ is compact, the kernel of $D$, the space of harmonic forms, is naturally identified with the real or complex ${ }^{1}$ cohomology of $M$ by the Hodge Theorem, and in this way one observes that the index of $D$ (with respect to the parity grading) is simply the Euler characteristic of $M$, whereas the index with respect to the other grading is the signature [AS3].

Now by Kasparov theory (good general references are [Bl] and [Hig1]), an elliptic operator such as $D$ gives rise to a $K$-homology class. In the case of a compact manifold, the index of the operator is recovered by looking at the image of this class under the map collapsing $M$ to a point. However, the $K$-homology class usually carries far more information than the index alone; for example, it determines the index of the operator with coefficients in any vector bundle, and even determines the families index in $K^{*}(X)$ of a family of twists of the operator, as determined by a vector bundle on $M \times X$. ( $X$ here is a parameter space.) When $M$ is non-compact, things are similar, except that usually there is no index, and the class lives in an

Received by the editors February 12, 1998.

1991 Mathematics Subject Classification. Primary 19K33; Secondary 19K35, 19K56, 58G12.

Key words and phrases. $K$-homology, de Rham operator, signature operator, Kasparov theory.

The author was partially supported by NSF Grant \# DMS-96-25336 and by the General Research Board of the University of Maryland.

${ }^{1}$ Depending on what scalars one is using. 
appropriate Kasparov group $K^{-*}\left(C_{0}(M)\right)$, which is locally finite $K$-homology, i.e., the relative group $K_{*}(\bar{M},\{\infty\})$, where $\bar{M}$ is the one-point compactification of $M .{ }^{2}$

In the case of the signature operator, the corresponding $K$-homology class (which lives in complex $K$-homology in degree $n=\operatorname{dim} M$ ) is a very rich object. Rationally, it is the Poincaré dual of the total $\mathcal{L}$-class (the Atiyah-Singer $L$-class, which differs from the Hirzebruch $L$-class only by certain well-understood powers of 2 ), but in addition, it also carries quite interesting integral information $[\mathrm{KM}],[\mathrm{R}],[\mathrm{RW}]$. It is therefore natural to ask if the $K$-homology class corresponding to the Euler characteristic operator carries any similar extra information (beyond the value of the Euler characteristic itself). We shall prove here that this is not the case. This result is supported by "popular wisdom," and of course one can prove a rational version of it by using the Atiyah-Singer Theorem and characteristic class calculations, but as far as I know the integral result has not been published before. (However, Bruce Williams has kindly shown me a purely topological proof, not any more elementary than the analytic one we shall give here, based on ideas in [BS, §6].)

We may as well work with real scalars and real $K$-theory, since triviality of the class in real $K$-homology will imply its triviality in complex $K$-homology as well. The fact that $D$ is odd with respect to the grading then gives us a class in $K O_{0}(M)$ (regardless of the value of $n$ ). Our main result is thus:

Theorem. Let $M^{n}$ be a complete Riemannian manifold, connected for simplicity, let $D$ be its Euler characteristic operator, and let $[D] \in K O_{0}(M)$ be its $K$-homology class (in locally finite $K$-homology). Then this class is as trivial as one can possibly expect it to be. In other words, if $M$ is compact, it is just $\chi(M) \in \mathbb{Z}$, with $\mathbb{Z}$ embedded in $K O_{0}(M)$ as the image of $K O_{0}(p t)$ under inclusion of a basepoint, and if $M$ is non-compact, the class is zero.

Before getting to the proof, we need two technical results. The first says that the $K$-homology class of the Euler characteristic (or signature) operator depends only on the manifold and not on the choice of a Riemannian metric.

Proposition. If $M^{n}$ is a connected manifold without boundary, either compact or non-compact, then the class of the Euler characteristic operator $[D] \in K O_{0}(M)$ is independent of the choice of a complete Riemannian metric on $M$. The same statement holds for the class of the signature operator in $K_{n}(M)$.

Proof (Sketch). This was proven in [Hig2], which was unfortunately never published, though some of the ideas appear in [Hig3] and [Hig4]. A proof in the more complicated context of the signature operator on Lipschitz manifolds appears in [Hil1] (for the compact case) and in [Hil2, §2] (for the non-compact case). For the reader's convenience (and also because we will need a similar argument later), we summarize the idea of Hilsum's approach. This makes use of the Baaj-Julg "unbounded" version ([BJ] or $[\mathrm{Bl}, \S 17.11])$ of the definition of the Kasparov $K$ homology group $K O_{0}(M)$. A class $[D]$ in $K O_{0}(M)$ is defined by an operator $D$ on a $\mathbb{Z} / 2$-graded Hilbert space $\mathcal{H}=\mathcal{H}^{+} \oplus \mathcal{H}^{-}$, where $\mathcal{H}$ is equipped with an action of

\footnotetext{
${ }^{2}$ Here $C_{0}(M)$ denotes continuous real- or complex-valued functions on $M$ vanishing at infinity, depending on whether one is using real or complex scalars. This algebra is contravariant in $M$, so a contravariant functor of $C_{0}(M)$ is covariant in $M$. Excision in Kasparov theory identifies $K^{-*}\left(C_{0}(M)\right)$ with $K^{-*}(C(\bar{M}), C(\mathrm{pt}))$, which is identified with relative $K$-homology. When $\bar{M}$ does not have finite homotopy type, $K$-homology here means Steenrod $K$-homology, as explained in $[\mathrm{KKS}]$.
} 
$C_{0}(M)$. We shall denote the action by multiplication, since in most cases of practical interest, $\mathcal{H}$ is a space of $L^{2}$ sections of a vector bundle over $M$, and $C_{0}(M)$ acts by multiplication operators. The operator $D$ and the action of $C_{0}(M)$ are required to satisfy the following properties:

(UB1) The operator $D$ is self-adjoint on $\mathcal{H}$ (though allowed to be unbounded).

(UB2) The operator $D$ is odd with respect to the grading of $\mathcal{H}$, i.e., $\operatorname{dom} D=$ $(\operatorname{dom} D)^{+} \oplus(\operatorname{dom} D)^{-}$, where $(\operatorname{dom} D)^{ \pm}=(\operatorname{dom} D) \cap \mathcal{H}^{ \pm}$, and $D$ maps $(\operatorname{dom} D)^{+}$to $\mathcal{H}^{-},(\operatorname{dom} D)^{-}$to $\mathcal{H}^{+}$.

(UB3) For a dense set of $f \in C_{0}(M), f$ maps the domain of $D$ into itself, and has bounded commutator with $D$.

(UB4) For $f \in C_{0}(M), f\left(1+D^{2}\right)^{-1}$ (which makes sense as a bounded operator, by the functional calculus for unbounded self-adjoint operators) is a compact operator.

The class $[D]$ only depends on the homotopy class of $D$ (within the space of all operators satisfying (UB1)-(UB4)), as made more precise in [BJ, Remarque 2.5(iv)]. In particular, if $a$ is bounded and self-adjoint, and if $t>0$, then $D, t D$, and $D+a$ all define the same class [D] [BJ, Remarque 2.5(iii)].

Given these preliminaries, the proof of the proposition is based on the following observations:

1. The space of complete Riemannian metrics on $M$ is arcwise connected, in fact is a convex cone (cf. [Hil2, Corollaire 1.4]).

2. If one joins two complete Riemannian metrics $g_{0}$ and $g_{1}$ by an arc $g_{t}$ of such metrics, then the corresponding Hodge $*$-operators vary continuously, so one gets a continuous field of Hilbert spaces $\mathcal{H}_{t}$ of $L^{2}$ forms. One can write down continuously varying explicit unitary operators $U_{t}: \mathcal{H}_{0} \rightarrow \mathcal{H}_{t}$ that can be used to carry operators on $\mathcal{H}_{t}$ back to $\mathcal{H}_{0}$.

3. If $D_{t}$ is the de Rham operator on $\mathcal{H}_{t}$, then it satisfies axioms (UB1)-(UB4) (with respect to either the parity grading or, when $n$ is even, the signature grading).

4. The operators $U_{t}^{*} D_{t} U_{t}$ satisfy the axioms for a homotopy of unbounded Kasparov modules. Hence $\left[D_{t}\right]$ is independent of $t$.

The details may be found in the references cited above.

Lemma 1. Let $M$ be a connected manifold without boundary, either compact with $\chi(M)=0$, or else non-compact. Then $M$ admits an everywhere non-vanishing vector field $X$ and a complete Riemannian metric $g$ with the properties that $\|X\|=1$ and $\left\|\nabla_{V} X\right\| \leq 1$ (pointwise everywhere), for all unit tangent vectors $V$. (All norms are taken with respect to $g$, and as usual, $\nabla$ denotes the covariant derivative with respect to the Riemannian connection.)

Proof. The condition that either $\chi(M)=0$ or else $M$ is non-compact guarantees that $M$ admits an everywhere non-vanishing vector field $X$. In the compact case, choose any Riemannian metric $g^{\prime}$ on $M$, and rescale $X$ to have length 1 everywhere. By compactness, $\|\nabla X\|^{\prime} \leq C$ for some constant $C>0$. (Here $\|\cdot\|^{\prime}$ denotes length with respect to $g^{\prime}$.) If we rescale $g^{\prime}$ to a metric $g$, multiplying vector lengths by $C$, then $\frac{1}{C} X$ has unit length in the new metric. Then if $V$ is any unit tangent vector with respect to $g^{\prime}, \frac{1}{C} V$ is a unit tangent vector in the new metric $g$, and

$$
\left\|\nabla_{\frac{1}{C} V}\left(\frac{1}{C} X\right)\right\|=\frac{1}{C^{2}}\left\|\nabla^{\prime}{ }_{V} X\right\|=\frac{C}{C^{2}}\left\|\nabla^{\prime}{ }_{V} X\right\|^{\prime} \leq 1 .
$$


For the non-compact case, we use the same idea, except we need to allow more general conformal rescaling. As in the compact case, we start with some complete Riemannian metric $g^{\prime}$ on $M$, and rescale $X$ to have length 1 everywhere (with respect to $g^{\prime}$ ). We then take $\langle\cdot, \cdot\rangle_{g}=e^{2 u}\langle\cdot, \cdot\rangle_{g^{\prime}}$, where the function $u$ is still to be determined. Then $e^{-u} X$ will be a unit vector field in the new metric $g$. If $V$ is a unit tangent vector with respect to $g^{\prime}$, then $e^{-u} V$ is a unit tangent vector with respect to $g$, and we have

$$
\begin{aligned}
\left\|\nabla_{e^{-u} V} e^{-u} X\right\| & =e^{-u}\left\|\nabla_{V} e^{-u} X\right\| \\
& =e^{-u}\left\|\left(V \cdot e^{-u}\right) X+e^{-u} \nabla_{V} X\right\| \\
& =\left\|e^{-u}(-V \cdot u) X+e^{-u} \nabla_{V} X\right\|^{\prime}
\end{aligned}
$$

(since $e^{-u}\|\|=\|\|^{\prime}$ )

$$
\begin{aligned}
=e^{-u} \| & -(V \cdot u) X+\nabla^{\prime}{ }_{V} X+(V \cdot u) X+(X \cdot u) V \\
& -\langle V, X\rangle^{\prime} \operatorname{grad}^{\prime}(u) \|^{\prime}
\end{aligned}
$$

(by the calculation in [LM, Proof of Theorem II.5.24, p. 133])

$$
\begin{aligned}
& =e^{-u}\left\|\nabla^{\prime}{ }_{V} X+(X \cdot u) V-\langle V, X\rangle^{\prime} \operatorname{grad}^{\prime}(u)\right\|^{\prime} \\
& \leq e^{-u}\left(\left\|\nabla^{\prime}{ }_{V} X\right\|^{\prime}+2\left\|\operatorname{grad}^{\prime}(u)\right\|^{\prime}\right),
\end{aligned}
$$

with $\operatorname{grad}^{\prime}(u)$ here computed in the original metric $g^{\prime}$. We just need to choose $u$ so that the right-hand side of $(1)$ is $\leq 1$.

To see that this is feasible, we fix a basepoint $x_{0}$ and choose $u$ to depend roughly only on the distance $r$ to $x_{0}$, with respect to the metric $g^{\prime}$. (Since the distance function may not be smooth, some smoothing may be required, but this does not affect the basic estimates.) We choose $u$ so that

$$
e^{u\left(r_{0}\right)} \geq 2 \max _{r \leq r_{0}}\left\|\nabla^{\prime} X\right\|^{\prime} .
$$

This bounds the first term in (1) by $\frac{1}{2}$. The second term is less of a problem, since $e^{-u}\left\|\operatorname{grad}^{\prime}(u)\right\|^{\prime}$ behaves like

$$
\left|\frac{d}{d r}\left(e^{-u(r)}\right)\right|
$$

Since we may choose $u$ to increase to $+\infty, e^{-u} \searrow 0$, and thus if $u$ is chosen smooth enough, the derivative of $e^{-u(r)}$ tends to 0 as $r \rightarrow \infty$. The new metric is complete since distances are bigger than in the old metric.

Proof of the Theorem. First observe that it is enough to prove the theorem in the non-compact case. For if $M$ is compact and connected, choose a basepoint $x_{0} \in M$ and let $N=M \backslash\left\{x_{0}\right\}$. Then $K O_{0}(N)=K O_{0}\left(M,\left\{x_{0}\right\}\right.$ ) (recall we are using locally finite homology), and one can show that $\left[D_{M}\right]$ maps to $\left[D_{N}\right]$ in the relative group. (This is not totally trivial, as the metric on $M$ has to be rescaled on $N$ in order to give a complete metric there, but see [Hig2] and [Hig3]. The point is that the $K$-homology class in locally finite homology really only sees the restrictions of the metric to compact sets, where all metrics are equivalent.) So if $\left[D_{N}\right]=0$ in $K O_{0}(N),\left[D_{M}\right]$ must come from $K O_{0}(\mathrm{pt})$, as claimed.

Thus we may assume $M$ is non-compact, and we will show $[D]=0$. (The same argument would show directly that $[D]=0$ if $M$ is compact with vanishing Euler 
characteristic.) The proposition says we are free to choose a complete metric on $M$ as we please. So apply Lemma 1 to choose a complete metric on $M$ and a vector field $X$ of length 1 everywhere with respect to the given metric, so that in addition $\left\|\nabla_{V} X\right\| \leq 1$ for all unit tangent vectors $V$. We identify the form bundle of $M$ with the Clifford algebra bundle Cliff $(T M)$ of the tangent bundle, with its standard grading in which vector fields are sections of $\operatorname{Cliff}(T M)^{-}$, and $D$ with the Dirac operator on Cliff $(T M) .{ }^{3}$ (This is legitimate by [LM, II, Theorem 5.12].) Let $A$ be the operator on $\operatorname{Cliff}(T M)$ defined by right Clifford multiplication by $X$ on $\operatorname{Cliff}(T M)^{+}$(the even part of Cliff $(T M)$ ) and by right Clifford multiplication by $-X$ on $\operatorname{Cliff}(T M)^{-}$(the odd part). We use right Clifford multiplication since it commutes with the symbol of $D$. Observe that, with respect to the $L^{2}$ inner product on sections of $\operatorname{Cliff}(T M), A$ is self-adjoint with square 1 . Furthermore, $A$ is odd with respect to the grading and commutes with multiplication by scalar-valued functions.

For $\lambda \geq 0$, let $D_{\lambda}=D+\lambda A$. Since $D$ and $A$ satisfy (UB1) and (UB2), so does each $D_{\lambda}$. Since $A$ is bounded, $D_{\lambda}$ also satisfies (UB3) and (UB4), and as noted above, all the $D_{\lambda}$ define the same Kasparov class.

Lemma 2. Let the metric on $M^{n}$ and the vector field $X$ satisfy the conclusions of Lemma 1, and let $A$ and $D_{\lambda}$ be as above. Then in the sense of ordering of self-adjoint operators,

$$
-n \lambda \leq D_{\lambda}^{2}-\left(D^{2}+\lambda^{2}\right) \leq n \lambda
$$

In particular, for $\lambda>n, D_{\lambda}^{2} \geq \lambda(\lambda-n)$, so $D_{\lambda}$ has a bounded inverse.

Proof of Lemma 2. Let $\omega$ be a section of $\operatorname{Cliff}(T M)$, say of $\operatorname{Cliff}(T M)^{+}$. Then if . denotes Clifford multiplication, we have:

$$
\begin{aligned}
D_{\lambda}^{2} \omega & =(D+\lambda A)^{2} \omega \\
& =(D+\lambda A)(D \omega+\lambda \omega \cdot X) \\
& =D^{2} \omega+\lambda D(\omega \cdot X)-\lambda(D \omega) \cdot X-\lambda^{2} \omega \cdot X \cdot X \\
& =\left(D^{2}+\lambda^{2}\right) \omega+\lambda(D(\omega \cdot X)-(D \omega) \cdot X) .
\end{aligned}
$$

However, from the definition of $D$ in terms of a local orthonormal frame $e_{1}, \ldots, e_{n}$, we have:

$$
D(\omega \cdot X)=\sum_{j} e_{j} \cdot \nabla_{e_{j}}(\omega \cdot X)
$$

which, since each $\nabla_{e_{j}}$ is a derivation, becomes

$$
\begin{aligned}
& =\sum_{j} e_{j} \cdot\left(\nabla_{e_{j}} \omega \cdot X+\omega \cdot \nabla_{e_{j}} X\right) \\
& =D(\omega) \cdot X+\sum_{j} e_{j} \cdot \omega \cdot \nabla_{e_{j}} X .
\end{aligned}
$$

Substituting this into equation (1), we obtain (locally)

$$
D_{\lambda}^{2} \omega=\left(D^{2}+\lambda^{2}\right) \omega+\lambda \sum_{j} e_{j} \cdot \omega \cdot \nabla_{e_{j}} X .
$$

\footnotetext{
${ }^{3}$ Since sign conventions differ, we emphasize that, for us, unit tangent vectors on $M$ have square -1 in the Clifford algebra.
} 
Taking the inner product with $\omega$, we obtain:

$$
\left\|D_{\lambda} \omega\right\|^{2}=\|D \omega\|^{2}+\lambda^{2}\|\omega\|^{2}+\lambda \sum_{j}\left\langle e_{j} \cdot \omega \cdot \nabla_{e_{j}} X, \omega\right\rangle,
$$

so estimating the last term, using the fact that $\left\|\nabla_{e_{j}} X\right\| \leq 1$, gives

$$
\left|\left\langle\left(D_{\lambda}^{2}-D^{2}-\lambda^{2}\right) \omega, \omega\right\rangle\right| \leq n \lambda\|\omega\|^{2} .
$$

The conclusion of the lemma follows. A similar argument applies if $\omega$ is a section of Cliff $(T M)^{-}$; the sign of the error term in equation (2) is reversed, but equation (3) remains unchanged.

Proof of Theorem (continued). We have seen that the class $[D]$ may be defined by $D_{\lambda}$, for any $\lambda>0$. In the "bounded picture" of Kasparov theory, the corresponding operator is

$$
\begin{aligned}
B_{\lambda} & =D_{\lambda}\left(1+D_{\lambda}^{2}\right)^{-\frac{1}{2}} \\
& =\frac{1}{\lambda} D_{\lambda}\left(\frac{1}{\lambda^{2}}+\frac{1}{\lambda^{2}} D_{\lambda}^{2}\right)^{-\frac{1}{2}} .
\end{aligned}
$$

The axioms satisfied by this operator which correspond to (UB1)-(UB4) are the following:

(B1) It is self-adjoint, of norm $\leq 1$.

(B2) It is odd with respect to the grading of $\operatorname{Cliff}(T M)$.

(B3) For $f \in C_{0}(M), f B_{\lambda} \sim B_{\lambda} f$ and $f B_{\lambda}^{2} \sim f$, where $\sim$ denotes equality modulo compact operators.

We claim $B_{\lambda} \rightarrow A$ in the strong operator topology as $\lambda \rightarrow \infty$. For this we apply Lemma 2. Indeed, for any $\omega$ :

$$
\begin{aligned}
\left\|B_{\lambda} \omega-A \omega\right\| & =\left\|\left(\frac{1}{\lambda^{2}}+\frac{1}{\lambda^{2}} D_{\lambda}^{2}\right)^{-\frac{1}{2}}\left(A+\frac{1}{\lambda} D\right) \omega-A \omega\right\| \\
\leq & \left\|\left(\frac{1}{\lambda^{2}}+\frac{1}{\lambda^{2}} D_{\lambda}^{2}\right)^{-\frac{1}{2}}\left(\frac{1}{\lambda} D \omega\right)\right\| \\
& +\left\|\left[\left(\frac{1}{\lambda^{2}}+\frac{1}{\lambda^{2}} D_{\lambda}^{2}\right)^{-\frac{1}{2}}-1\right] A \omega\right\| .
\end{aligned}
$$

To estimate the two terms on the right, note that by (3) we have:

$$
1+\lambda^{2}-C \lambda \leq 1+D_{\lambda}^{2} \leq 1+D^{2}+\lambda^{2}+C \lambda .
$$

In particular, as $\lambda \rightarrow \infty$,

$$
\left\|\left(\frac{1}{\lambda^{2}}+\frac{1}{\lambda^{2}} D_{\lambda}^{2}\right)^{-\frac{1}{2}}\right\| \leq 1+O\left(\frac{1}{\lambda}\right) .
$$

Since also $\frac{1}{\lambda} D \omega \rightarrow 0$, the first term on the right in (4) goes to 0 . To estimate the second term in (4), note that on the spectral subspace where $0 \leq D^{2} \leq \mu$, we have

$$
1+\lambda^{2}-C \lambda \leq 1+D_{\lambda}^{2} \leq 1+\mu+\lambda^{2}+C \lambda,
$$


and thus

$$
1-O\left(\frac{1}{\lambda}\right) \leq\left(\frac{1}{\lambda^{2}}+\frac{1}{\lambda^{2}} D_{\lambda}^{2}\right)^{-\frac{1}{2}} \leq 1+O\left(\frac{1}{\lambda}\right) .
$$

Hence the second term in (4) goes to 0 for $A \omega$ in the spectral subspace where $0 \leq$ $D^{2} \leq \mu$. Since we can let $\mu \rightarrow \infty$, we see $B_{\lambda} \rightarrow A$ in the strong operator topology. Since $B_{\lambda}$ and $A$ each satisfy the conditions (B1)-(B2), we have a candidate for a homotopy of Kasparov modules between the Kasparov modules defined by $D$ and by $A$. But $A=A^{*}=A^{-1}$ and $A$ commutes with the action of $C_{0}(M)$. In other words, in the case of $A$, we have a degenerate module, in the sense that we can replace $\sim$ by $=$ in condition (B3). Hence the class $[A]$ is trivial in $K O_{0}(M)$.

So we will be done if we can check the remaining conditions for a homotopy of Kasparov modules. There are only two of these. First of all, we need to check that $1-B_{\lambda}^{2} \rightarrow 1-A^{2}=0$ in norm, not just strongly. But $1-B_{\lambda}^{2}=\left(1+D_{\lambda}^{2}\right)^{-1}$, which is bounded in norm by $\left(\lambda^{2}-n \lambda\right)^{-1}$ for large $\lambda$, by Lemma 2 . Finally, we need to check that for $f \in C_{0}(M)$, the commutator of $f$ and $B_{\lambda}$ tends to 0 in norm. For this, take $f$ smooth so that $[D, f]$ is bounded, and estimate as follows:

$$
\left[f, B_{\lambda}\right]=\left[f, D_{\lambda}\left(1+D_{\lambda}^{2}\right)^{-1 / 2}\right]=\left[f, D_{\lambda}\right]\left(1+D_{\lambda}^{2}\right)^{-1 / 2}+D_{\lambda}\left[f,\left(1+D_{\lambda}^{2}\right)^{-1 / 2}\right] .
$$

Now, $\left[f, D_{\lambda}\right]=[f, D]$ and $\left(1+D_{\lambda}^{2}\right)^{-1 / 2} \rightarrow 0$ in norm, so the first term on the right goes to 0 in norm. As for the second term, we have (following [Bl, p. 199])

$$
D_{\lambda}\left[f,\left(1+D_{\lambda}^{2}\right)^{-1 / 2}\right]=\frac{1}{\pi} \int_{0}^{\infty} \mu^{-1 / 2} D_{\lambda}\left[f,\left(1+D_{\lambda}^{2}+\mu\right)^{-1}\right] d \mu,
$$

and

$$
D_{\lambda}\left[f,\left(1+D_{\lambda}^{2}+\mu\right)^{-1}\right]=D_{\lambda}\left(1+D_{\lambda}^{2}+\mu\right)^{-1}\left[1+D_{\lambda}^{2}+\mu, f\right]\left(1+D_{\lambda}^{2}+\mu\right)^{-1} .
$$

Now use the fact that

$$
\left[1+D_{\lambda}^{2}+\mu, f\right]=\left[D_{\lambda}^{2}, f\right]=D_{\lambda}\left[D_{\lambda}, f\right]+\left[D_{\lambda}, f\right] D_{\lambda}=D_{\lambda}[D, f]+[D, f] D_{\lambda} .
$$

We obtain that

$$
\begin{aligned}
D_{\lambda}[f & \left.\left(1+D_{\lambda}^{2}+\mu\right)^{-1}\right] \\
& =\frac{D_{\lambda}^{2}}{1+D_{\lambda}^{2}+\mu}[D, f] \frac{1}{1+D_{\lambda}^{2}+\mu}+\frac{D_{\lambda}}{1+D_{\lambda}^{2}+\mu}[D, f] \frac{D_{\lambda}}{1+D_{\lambda}^{2}+\mu},
\end{aligned}
$$

which can be bounded in norm by $2\|[D, f]\|$ times $\left(1+\lambda^{2}-n \lambda+\mu\right)^{-1}$, for large $\lambda$. But

$$
\frac{1}{\pi} \int_{0}^{\infty} \mu^{-1 / 2} \frac{1}{1+\lambda^{2}-n \lambda+\mu} d \mu=\frac{1}{\sqrt{1+\lambda^{2}-n \lambda}} \rightarrow 0 \text { as } \lambda \rightarrow 0 .
$$

So $\left\|\left[f, B_{\lambda}\right]\right\| \rightarrow 0$ and we have a homotopy of Kasparov modules. Thus $[D]=[A]=$ 0 .

\section{REFERENCES}

[AS3] M. F. Atiyah and I. M. Singer, The index of elliptic operators, III, Ann. of Math. (2) 87 (1968), 546-604. MR 38:5245

[BJ] S. Baaj and P. Julg, Théorie bivariante de Kasparov et opérateurs non bornés dans les $C^{*}$-modules hilbertiens, C. R. Acad. Sci. Paris Sér. I Math. 296 (21) (1983), 875-878. MR 84m: 46091

[Bl] B. Blackadar, K-Theory for Operator Algebras, Math. Sci. Res. Inst. Publ., vol. 5, SpringerVerlag, New York, Berlin, 1986. MR 88g:46082 
[BS] J. C. Becker and R. E. Schultz, The real semicharacteristic of a fibered manifold, Quart. J. Math. Oxford (2) 33 (1982), 385-403. MR 84a:57022

[Ga] M. P. Gaffney, A special Stokes's theorem for complete Riemannian manifolds, Ann. of Math. (2) 60 (1954), 140-145. MR 15:986d

[Hig1] N. Higson, A primer on KK-theory, Operator theory: operator algebras and applications, Part 1 (Durham, NH, 1988) (W. Arveson and R. Douglas, eds.), Proc. Sympos. Pure Math., vol. 51, Part 1, Amer. Math. Soc., Providence, RI, 1990, pp. 239-283. MR 92g:19005

[Hig2] N. Higson, K-homology and operators on non-compact manifolds, Unpublished preprint, ca. 1989.

[Hig3] N. Higson, A note on the cobordism invariance of the index, Topology 30 (3) (1991), 439-443. MR 92f:58171

[Hig4] N. Higson, On the K-theory proof of the index theorem, Index theory and operator algebras (Boulder, CO, 1991), Contemp. Math., vol. 148, Amer. Math. Soc., Providence, RI, 1993, pp. 67-86. MR 95a:19009

[Hil1] M. Hilsum, Signature operator on Lipschitz manifolds and unbounded Kasparov bimodules, Operator algebras and their connections with topology and ergodic theory (Buşteni, 1983), Lecture Notes in Math., vol. 1132, Springer-Verlag, Berlin, New York, 1985, pp. 254-288. MR 87d:58133

[Hil2] M. Hilsum, Fonctorialité en $K$-théorie bivariante pour les variétés lipschitziennes, $K$ Theory 3 (5) (1989), 401-440. MR 91j:19012

[KKS] D. S. Kahn, J. Kaminker, and C. Schochet, Generalized homology theories on compact metric spaces, Michigan Math. J. 24 (2) (1977), 203-224. MR 57:13921

$[\mathrm{KM}]$ J. Kaminker and J. G. Miller, Homotopy invariance of the analytic index of signature operators over $C^{*}$-algebras, J. Operator Theory 14 (1) (1985), 113-127. MR 87b:58082

[LM] H. B. Lawson, Jr. and M.-L. Michelsohn, Spin Geometry, Princeton Mathematical Ser., vol. 38, Princeton Univ. Press, Princeton, NJ, 1989. MR 91g:53001

[R] J. Rosenberg, Analytic Novikov for topologists, Novikov Conjectures, Index Theorems and Rigidity, vol. 1 (S. Ferry, A. Ranicki, and J. Rosenberg, eds.), London Math. Soc. Lecture Notes, vol. 226, Cambridge Univ. Press, Cambridge, 1995, pp. 338-372. MR 97b:58138

[RW] J. Rosenberg and S. Weinberger, The signature operator at 2, In preparation.

Department of Mathematics, University of Maryland, College Park, Maryland 20742

E-mail address: jmr@math.umd.edu 